\title{
Looking towards the rim of the active site cavity of druggable human Carbonic Anhydrase isoforms
}

\author{
Francesca Mancuso, ${ }^{\mathrm{a}}$ Anna Di Fiore, ${ }^{\mathrm{b}}$ Laura De Luca, ${ }^{\mathrm{a}}$ Andrea Angeli, ${ }^{\mathrm{c}}$ Simona M. Monti, ${ }^{\mathrm{b}}$ \\ Giuseppina De Simone, ${ }^{\mathrm{b}}$ Claudiu T. Supuran, ${ }^{\mathrm{c}}$ Rosaria Gitto ${ }^{\mathrm{a}^{*}}$ \\ aDipartimento di Scienze Chimiche, Biologiche, Farmaceutiche ed Ambientali (CHIBIOFARAM), Università degli \\ Studi di Messina, Viale Palatucci, Polo Didattico SS. Annunziata, 98168, Messina, Italy \\ 'Istituto di Biostrutture e Bioimmagini-CNR, Via Mezzocannone 16, 80134, Napoli, Italy \\ 'Dipartimento NEUROFARBA, Università di Firenze, Via Ugo Schiff 6, 50019 - Sesto Fiorentino, Italy
}

\section{Content:}

1. Experimental Section

\subsection{Chemistry}

1.2. CA Inhibition Assay

1.3. X-ray crystallographic studies

1.4. Docking studies

2. $\quad$ Selected representative ${ }^{1} \mathrm{H}$ and ${ }^{13} \mathrm{C}$ NMR spectra

3. Table S1

\section{Experimental Section}

\subsection{Chemistry}

All reagents were used without further purification and bought from common commercial suppliers (Sigma-Aldrich Milan, Italy; Alfa Aesar Karlsruhe, Germany). Melting points were determined on a Buchi B-545 apparatus (BUCHI Labortechnik AG Flawil, Switzerland) and are uncorrected. By combustion analysis $(\mathrm{C}, \mathrm{H}, \mathrm{N})$ carried out on a Carlo Erba Model 1106-Elemental Analyzer we determined the purity of synthesized compounds; the results confirmed a $\geq 95 \%$ purity. Merck Silica Gel 60 F254 plates were used for analytical TLC (Merck KGaA, Darmstadt, Germany). For detection, iodine vapor and UV light $(254 \mathrm{~nm})$ were used. ${ }^{1} \mathrm{H}-\mathrm{NMR}$ and ${ }^{13} \mathrm{C}-\mathrm{NMR}$ spectra were measured in dimethylsulfoxide-d6 (DMSO-d $_{\text {) }}$ with a Varian Gemini 300 spectrometer (Varian Inc. Palo Alto, California USA); chemical shifts are expressed in $\delta(\mathrm{ppm})$ and coupling constants $(J)$ in hertz. All exchangeable protons were confirmed by addition of $\mathrm{D}_{2} \mathrm{O}$. $R_{\underline{\mathrm{f}}}$ values were determined on TLC plates using a mixture of DCM/MeOH (96/4) as eluent.

\section{General procedures for the synthesis preparation of phenyl(piperazin-1-yl)methanone derivatives $4 a-p$}

To a stirred solution of 1-Boc-piperazine (1 mmol) in DCM (3 mL), EDIPA (1.5 molar equivalents) and the appropriate benzoyl chloride derivative (3a-p) (1 molar equivalent) were added. The reaction mixture was stirred for different reaction times (ranging from 3-4 hours) at room temperature and monitored by TLC until the disappearance of the starting materials. Thus, TFA ( 8 molar equivalents) was added at $0^{\circ} \mathrm{C}$ and the resulting mixture was stirred for 3 hours at $25{ }^{\circ} \mathrm{C}$. After the reaction was completed it was cooled into ice and diluted with DCM $(2 \mathrm{~mL})$ and $2 \mathrm{M} \mathrm{K}_{2} \mathrm{CO}_{3}(2 \mathrm{~mL})$. The mixture was extracted with DCM $(3 \times 5 \mathrm{~mL})$, the combined organic layers were dried over $\mathrm{Na}_{2} \mathrm{SO}_{4}$, filtered, 
and concentrated under vacuo. The residue was purified by treatment with $\mathrm{Et}_{2} \mathrm{O}$ giving the desired known intermediates $\mathbf{4 a - p}$, some of them are commercially available. The registered CAS numbers for resulting compounds $\mathbf{4 a - p}$ have been already assigned as reported below.

\begin{tabular}{|l|l|}
\hline cpd & CAS number \\
\hline 4a & $13754-38-6$ \\
\hline $\mathbf{4 b}$ & $139516-64-6$ \\
\hline $\mathbf{4 c}$ & $179334-10-2$ \\
\hline $\mathbf{4 d}$ & $102391-98-0$ \\
\hline $\mathbf{4 e}$ & $13754-45-5$ \\
\hline $\mathbf{4 f}$ & $100939-90-0$ \\
\hline $\mathbf{4 g}$ & $54042-47-6$ \\
\hline $\mathbf{4 h}$ & $926202-11-1$ \\
\hline
\end{tabular}

\begin{tabular}{|l|l|}
\hline cpd & CAS number \\
\hline $\mathbf{4 i}$ & $1016819-18-3$ \\
\hline $\mathbf{4 j}$ & $59939-72-9$ \\
\hline $\mathbf{4 k}$ & $100939-88-6$ \\
\hline $\mathbf{4 l}$ & $100939-89$ \\
\hline $\mathbf{4 m}$ & $94747-49-6$ \\
\hline $\mathbf{4 n}$ & $885101-42-8$ \\
\hline $\mathbf{4 o}$ & $341529-34-8$ \\
\hline $\mathbf{4 p}$ & $72141-41-4$ \\
\hline
\end{tabular}

General procedures for the synthesis of 4-(4-aroylpiperazine-1carbonyl)benzenesulfonamide derivatives (5a-s)

To a solution of 4-(aminosulfonyl)benzoic acid (1 mmol) dissolved in dimethylformamide (DMF) (2 mL) N,N,N',N'-tetramethyl-O-(1H-benzotriazol-1-yl)-uraniumhexafluorophosphate (HBTU) (1 molar equivalent) was added. The mixture was stirred at room temperature for $1 \mathrm{~h}$. Then, TEA (2 molar equivalents) and appropriate 4-benzoylpiperazine derivatives 4a-p ( 1 molar equivalent) were added. The reaction mixture was left overnight at room temperature and then quenched with $\mathrm{H}_{2} \mathrm{O}$ (10 $\mathrm{mL})$ and extracted with EtOAc $(3 \times 10 \mathrm{~mL})$. The organic phase was washed with saturated $\mathrm{NaCl}$ solution, dried with $\mathrm{Na}_{2} \mathrm{SO}_{4}$ and concentrated until dryness under reduced pressure. The residue was purified by crystallization from $\mathrm{Et}_{2} \mathrm{O}$ and $\mathrm{EtOH}$ to give the desired final compounds 5a-p as white powder.

To a suspension of the nitro derivatives 5n-p (1mmol) and a catalytic amount of $\mathrm{Pd} / \mathrm{C}$ in EtOH (15 $\mathrm{mL}$ ), hydrazine hydrate (10 molar equivalents) was slowly added. The reaction was stirred and refluxed $\left(70{ }^{\circ} \mathrm{C}\right)$ under nitrogen atmosphere. Then, the mixture was filtered through celite, the cake was later washed with EtOAc. The solution was evaporated in vacuo to give the crude product, then dissolved in EtOAc and washed with $\mathrm{H}_{2} \mathrm{O}\left(3 \times 10 \mathrm{~mL}\right.$ ). The organic phase was dried with $\mathrm{Na}_{2} \mathrm{SO}_{4}$ and concentrated until dryness. The residue was purified by crystallization from $\mathrm{Et}_{2} \mathrm{O}$ and $\mathrm{EtOH}$ to give the title compounds $\mathbf{5 q} \mathbf{q}$.

\section{4-(4-Benzoylpiperazine-1-carbonyl)benzenesulfonamide 5a}

Yield: 45\%; M.p.: $248-249^{\circ} \mathrm{C}$; $\mathrm{R}_{f}=0.24 .{ }^{1} \mathrm{H}-\mathrm{NMR}$ (DMSO- $\left.d_{6}\right)$ : $(\delta) 3.55-3.93\left(\mathrm{~m}, 8 \mathrm{H}, \mathrm{CH}_{2}\right), 7.40-$ 7.47 (m, 5H, ArH), 7.50 (s, 2H, NH$), 7.62$ (d, J=8.2, 2H, ArH ), 7.89 (d, J=8.2, 2H, ArH); ${ }^{13} \mathrm{C}-\mathrm{NMR}$ (DMSO-d6): 42.13, 47.71, 125.64, 126.99, 127.65, 128.33, 129.64, 135.45, 138.62, 144.91, 169.33, 171.66. Anal. for $\left(\mathrm{C}_{18} \mathrm{H}_{19} \mathrm{~N}_{3} \mathrm{O}_{4} \mathrm{~S}\right)$ : C 57.89\%, H 5.13\%, N 11.25\%; Found: C 57.99\%, H 5.23\%, N $11.35 \%$.

\section{4-[4-(2-Fluorobenzoyl)piperazine-1-carbonyl]benzenesulfonamide, $\mathbf{5} \mathbf{b}$}

Yield: 72\%; M.p.: 268-269 ${ }^{\circ} \mathrm{C} ; \mathrm{R}_{f}=0.12 .{ }^{1} \mathrm{H}-\mathrm{NMR}$ (DMSO-d6): $(\delta)$ 3.55-3.76 (m, $\left.\mathrm{CH}_{2}, 8 \mathrm{H}\right), 7.31-$ $7.44(\mathrm{~m}, 4 \mathrm{H}, \mathrm{ArH}), 7.49\left(\mathrm{~s}, 2 \mathrm{H}, \mathrm{NH}_{2}\right), 7.57-7.65(\mathrm{~m}, 2 \mathrm{H}, \mathrm{ArH}), 7.88-7.95$ (m, 2H, ArH). Anal. for $\left(\mathrm{C}_{18} \mathrm{H}_{18} \mathrm{FN}_{3} \mathrm{O}_{4} \mathrm{~S}\right)$ : C 55.23\%, H 4.64\%, N 10.74\%; Found: C 55.33\%, H 4.74\%, N 10.54\%. 
4-[4-(3-Fluorobenzoyl)piperazine-1-carbonyl]benzenesulfonamide, $\mathbf{5 c}$

Yield: 43\%; M.p.: 214-215 ${ }^{\circ}$; $\mathrm{R}_{f}=0.11 .{ }^{1} \mathrm{H}-\mathrm{NMR}$ (DMSO-d6): $(\delta)$ 3.57-3.76 (m, 8H, $\left.\mathrm{CH}_{2}\right), 7.23-$ $7.33(\mathrm{~m}, 3 \mathrm{H}, \mathrm{ArH}), 7.49$ (s, 2H, NH 2$)$. 7.61-7.64 (m, 3H, ArH), 7.87-7.90 (m, 2H, ArH). Anal. for $\left(\mathrm{C}_{18} \mathrm{H}_{18} \mathrm{FN}_{3} \mathrm{O}_{4} \mathrm{~S}\right): \mathrm{C} 55.23 \%, \mathrm{H} 4.64 \%$, N 10.74\%; Found: C 55.03\%, H 4.44\%, N 10.54\%.

4-[4-(4-Fluorobenzoyl)piperazine-1-carbonyl]benzenesulfonamide, $\mathbf{5 d}$

Yield: 42\%; M.p.: 212-214 ${ }^{\circ} \mathrm{C}$; $\mathrm{R}_{f}=0.10 .{ }^{1} \mathrm{H}-\mathrm{NMR}$ (DMSO- $\left.d_{6}\right):(\delta) 3.03-3.40\left(\mathrm{~m}, 8 \mathrm{H}, \mathrm{CH}_{2}\right), 7.26-$ 7.33 (m, 1H, ArH), 7.49 (s, 2H, NH ), 7.60-7.74 (m, 2H, ArH), 7.83-8.01 (m, 4H, ArH), 8.11-8.14 $(\mathrm{m}, 1 \mathrm{H}, \mathrm{ArH})$. Anal. for $\left(\mathrm{C}_{18} \mathrm{H}_{18} \mathrm{FN}_{3} \mathrm{O}_{4} \mathrm{~S}\right)$ : C 55.23\%, $\mathrm{H} 4.64 \%, \mathrm{~N} 10.74 \%$ Found: $\mathrm{C} 55.13 \%, \mathrm{H}$ $4.54 \%, \mathrm{~N} 10.64 \%$.

4-[4-(2-Chlorobenzoyl)piperazine-1-carbonyl]benzenesulfonamide, $\mathbf{5 e}$ Yield: 63\%; M.p.: $249-250^{\circ} \mathrm{C} ; \mathrm{R}_{f}=0.15 .{ }^{1} \mathrm{H}-\mathrm{NMR}\left(\mathrm{DMSO}-d_{6}\right):(\delta) 3.16(\mathrm{~m}, 2 \mathrm{H}), 3.24(\mathrm{~m}, 2 \mathrm{H}), 3.67-$ $3.73(\mathrm{~m}, 4 \mathrm{H}), 7.43(\mathrm{~m}, 4 \mathrm{H}, \mathrm{ArH}), 7.50\left(\mathrm{~s}, 2 \mathrm{H}, \mathrm{NH}_{2}\right), 7.62(\mathrm{~d}, J=8.2,2 \mathrm{H}, \mathrm{ArH}), 7.88(\mathrm{~d}, J=8.2,2 \mathrm{H}$, ArH). Anal. for $\left(\mathrm{C}_{18} \mathrm{H}_{18} \mathrm{ClN}_{3} \mathrm{O}_{4} \mathrm{~S}\right)$ : C 53.01\%, H 4.45\%, N 10.30\%; Found: C 52.91\%, H 4.35\%, N $10.20 \%$.

4-[4-(3-Chlorobenzoyl)piperazine-1-carbonyl]benzenesulfonamide, $\mathbf{5 f}$

Yield: 63\%; M.p.: $193-195^{\circ} \mathrm{C}$; $\mathrm{R}_{f}=0.15 .{ }^{1} \mathrm{H}-\mathrm{NMR}$ (DMSO- $\left.d_{6}\right)$ : $(\delta)$ 3.27-3.41 (m, 4H), 3.62-3.67 (m,

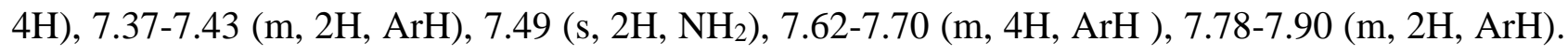
Anal. for $\left(\mathrm{C}_{18} \mathrm{H}_{18} \mathrm{ClN}_{3} \mathrm{O}_{4} \mathrm{~S}\right)$ : C 53.01\%, H 4.45\%, N 10.30\%; Found: C 53.11\%, H 4.55\%, N 10.40\%.

4-[4-(4-Chlorobenzoyl)piperazine-1-carbonyl]benzenesulfonamide, $\mathbf{5 g}$

Yield: 50\%; M.p.: 245-246 ${ }^{\circ}$; $\mathrm{R}_{f}=0.14 .{ }^{1} \mathrm{H}-\mathrm{NMR}$ (DMSO-d $)$ : $(\delta)$ 3.50-3.75 (m, 8H, $\mathrm{CH}_{2}$ ), 7.417.48 (m, 2H, ArH), 7.50 (s, 2H, NH 2$), 7.52-7.54$ (m, 2H, ArH ), 7.63 (d, J=8.2, 2H, ArH), 7.87 (d, $J=8.2,2 \mathrm{H}, \mathrm{ArH}$ ); ${ }^{13} \mathrm{C}-\mathrm{NMR}$ (DMSO-d $): 41.84,46.94,125.99,127.76,128.70,129.20,134.41$, 134.55, 138.87, 145.04, 168.20, 168.41. Anal. for $\left(\mathrm{C}_{18} \mathrm{H}_{18} \mathrm{ClN}_{3} \mathrm{O}_{4} \mathrm{~S}\right)$ : C 53.01\%, H 4.45\%, N 10.30\%; Found: C $53.21 \%, \mathrm{H} 4.65 \%, \mathrm{~N} 10.50 \%$.

\section{4-[4-(2-Bromobenzoyl)piperazine-1-carbonyl]benzenesulfonamide, $\mathbf{5 h}$}

Yield: 49\%; M.p.: 209-211 ${ }^{\circ} \mathrm{C}$; $\mathrm{R}_{f}=0.19 .{ }^{1} \mathrm{H}-\mathrm{NMR}$ (DMSO- $\left.d_{6}\right)$ : $(\delta)$ 3.22-3.40 (m, 4H), 3.69-3.74 (m,

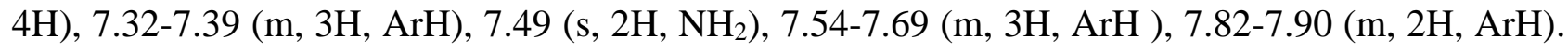
Anal. for $\left(\mathrm{C}_{18} \mathrm{H}_{18} \mathrm{BrN}_{3} \mathrm{O}_{4} \mathrm{~S}\right)$ : C 47.80\%, H 4.01\%, N 9.29\%; Found: C 47.68\%, H 4.21\%, N 9.41\%.

\section{4-[4-(3-Bromobenzoyl)piperazine-1-carbonyl]benzenesulfonamide, $\mathbf{5 i}$}

Yield: 86\%; M.p.: $193-194^{\circ} \mathrm{C} ; \mathrm{R}_{f}=0.18 .{ }^{1} \mathrm{H}-\mathrm{NMR}\left(\mathrm{DMSO}-d_{6}\right)$ : $(\delta)$ 3.52-3.87 (m, 8H, $\left.\mathrm{CH}_{2}\right), 7.38-43$ (m, 2H, ArH), $7.50\left(\mathrm{~s}, 2 \mathrm{H}, \mathrm{NH}_{2}\right), 7.59-7.68(\mathrm{~m}, 4 \mathrm{H}, \mathrm{ArH}), 7.83-7.92(\mathrm{~m}, 2 \mathrm{H}, \mathrm{ArH})$. Anal. for $\left(\mathrm{C}_{18} \mathrm{H}_{18} \mathrm{BrN}_{3} \mathrm{O}_{4} \mathrm{~S}\right): \mathrm{C} 47.80 \%, \mathrm{H} 4.01 \%, \mathrm{~N} 9.29 \%$; Found: C 47.98\%, H 4.11\%, N 9.39\%.

\section{4-[4-(4-Bromobenzoyl)piperazine-1-carbonyl]benzenesulfonamide, $\mathbf{5} \mathbf{j}$}

Yield: 47\%; M.p.: 222-224 ${ }^{\circ}$; $\mathrm{R}_{f}=0.18 .{ }^{1} \mathrm{H}-\mathrm{NMR}\left(\mathrm{DMSO}-d_{6}\right):(\delta) 3.51-3.75\left(\mathrm{~m}, 8 \mathrm{H}, \mathrm{CH}_{2}\right), 7.43-$ (m, 2H, ArH), 7.51 (s, 2H, NH 2$), 7.53-7.58$ (m, 2H, ArH ), 7.63-7.70 (m, 2H, ArH), 7.87-7.93 (m,

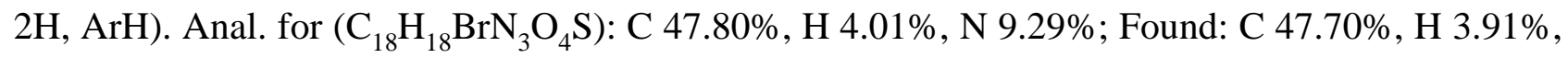
N $9.19 \%$. 
4-[4-(2-Methoxybenzoyl)piperazine-1-carbonyl]benzenesulfonamide, $\mathbf{5 k}$

Yield: 56\%; M.p.: 268-269 ${ }^{\circ} \mathrm{C} ; \mathrm{R}_{f}=0.13 .{ }^{1} \mathrm{H}-\mathrm{NMR}$ (DMSO- $\left.d_{6}\right):(\delta) 3.24\left(\mathrm{~m}, 4 \mathrm{H}, \mathrm{CH}_{2}\right), 3.57(\mathrm{~m}, 4 \mathrm{H}$, $\left.\mathrm{CH}_{2}\right), 3.90$ (s, 3H, $\left.\mathrm{OCH}_{3}\right), 6.98-7.38$ (m, 4H, ArH), 7.50 (s, 2H, NH 2$), 7.60-7.65$ (m, 2H, ArH). 7.79$7.86(\mathrm{~m}, 2 \mathrm{H}, \mathrm{ArH})$. Anal. for $\left(\mathrm{C}_{19} \mathrm{H}_{21} \mathrm{~N}_{3} \mathrm{O}_{5} \mathrm{~S}\right): \mathrm{C} 56.56 \%, \mathrm{H} 5.25 \%, \mathrm{~N} \mathrm{10.42 \%}$; Found: C 56.36\%, H $5.05 \%, \mathrm{~N} 10.22 \%$.

4-[4-(3-Methoxybenzoyl)piperazine-1-carbonyl]benzenesulfonamide, $\mathbf{5 1}$

Yield: 43\%; M.p.: $144-146^{\circ} \mathrm{C} ; \mathrm{R}_{f}=0.13 .{ }^{1} \mathrm{H}-\mathrm{NMR}$ (DMSO- $\left.d_{6}\right):(\delta)$ 3.36-3.63 (m, 4H, $\left.\mathrm{CH}_{2}\right), 3.77$ (s, $\left.3 \mathrm{H}, \mathrm{OCH}_{3}\right), 4.23-4.63\left(\mathrm{~m}, 4 \mathrm{H}, \mathrm{CH}_{2}\right), 6.95-7.03(\mathrm{~m}, 3 \mathrm{H}, \mathrm{ArH}), 7.45-7.49$ (m, 1H, ArH), 7.50 (s, 2H, $\left.\mathrm{NH}_{2}\right)$, 7.61-7.64 (m, 2H, ArH). 7.87-7.90 (m, 2H, ArH). Anal. for $\left(\mathrm{C}_{19} \mathrm{H}_{21} \mathrm{~N}_{3} \mathrm{O}_{5} \mathrm{~S}\right): \mathrm{C} 56.56 \%, \mathrm{H}$ $5.25 \%$, N 10.42\%; Found: C 56.36\%, H 5.05\%, N $10.22 \%$.

4-[4-(4-Methoxybenzoyl)piperazine-1-carbonyl]benzenesulfonamide, $\mathbf{5 m}$

Yield: 52\%; M.p.: 260-261 ${ }^{\circ} \mathrm{C} ; \mathrm{R}_{f}=0.12 .{ }^{1} \mathrm{H}-\mathrm{NMR}$ (DMSO-d $)$ : $(\delta)$ 3.34-3.47 (m, 4H, $\left.\mathrm{CH}_{2}\right), 3.56-3.68$ $\left(\mathrm{m}, 4 \mathrm{H}, \mathrm{CH}_{2}\right), 3.71\left(\mathrm{~s}, 3 \mathrm{H}, \mathrm{OCH}_{3}\right), 6.97-7.01(\mathrm{~m}, 2 \mathrm{H}, \mathrm{ArH}), 7.37-7.41(\mathrm{~m}, 2 \mathrm{H}, \mathrm{ArH}), 7.50(\mathrm{~s}, 2 \mathrm{H}$, $\mathrm{NH}_{2}$ ), 7.63-7.65 (d, $\left.J=8.2,2 \mathrm{H}, \mathrm{ArH}\right)$. 7.86-7.90 (d, $\left.J=8.2,2 \mathrm{H}, \mathrm{ArH}\right)$. Anal. for $\left(\mathrm{C}_{19} \mathrm{H}_{21} \mathrm{~N}_{3} \mathrm{O}_{5} \mathrm{~S}\right): \mathrm{C}$ $56.56 \%$, H 5.25\%, N 10.42\%; Found: C 56.46\%, H 5.15\%, N 10.32\%.

4-[4-(2-Nitrobenzoyl)piperazine-1-carbonyl]benzenesulfonamide, $\mathbf{5 n}$

Yield: 55\%; M.p.: $231-232^{\circ} \mathrm{C}$; $\mathrm{R}_{f}=0.16 .{ }^{1} \mathrm{H}-\mathrm{NMR}\left(\mathrm{DMSO}-d_{6}\right):(\delta) 3.37-3.77\left(\mathrm{~m}, 8 \mathrm{H}, \mathrm{CH}_{2}\right), 7.48(\mathrm{~m}$, 2H, ArH), 7.56-7.77 (m, 4H, ArH), 7.83-7.96 (m, 3H, ArH ), 8.20-8.25 (m, 1H, ArH). Anal. for $\left(\mathrm{C}_{18} \mathrm{H}_{18} \mathrm{~N}_{4} \mathrm{O}_{6} \mathrm{~S}\right)$ : C 51.67\%, H 4.34\%, N 13.39\%; Found: C 51.71\%, H 4.39\%, N 13.44\%.

4-[4-(3-Nitrobenzoyl)piperazine-1-carbonyl]benzenesulfonamide, 5o

Yield: 75\%; M.p.: 243-244 ${ }^{\circ} \mathrm{C}$; $\mathrm{R}_{f}=0.18$. ${ }^{1} \mathrm{H}-\mathrm{NMR}$ (DMSO- $\left.d_{6}\right):(\delta) 3.38-3.49\left(\mathrm{~m}, 4 \mathrm{H}, \mathrm{CH}_{2}\right), 3.65-$ $3.74\left(\mathrm{~m}, 4 \mathrm{H}, \mathrm{CH}_{2}\right), 7.45\left(\mathrm{~s}, 2 \mathrm{H}, \mathrm{NH}_{2}\right), 7.61-8.29(\mathrm{~m}, 8 \mathrm{H}, \mathrm{ArH})$. Anal. for $\left(\mathrm{C}_{18} \mathrm{H}_{18} \mathrm{~N}_{4} \mathrm{O}_{6} \mathrm{~S}\right): \mathrm{C} 51.67 \%$, H 4.34\%, N 13.39\%; Found: C 51.70\%, H 4.28\%, N $13.53 \%$.

4-[4-(4-Nitrobenzoyl)piperazine-1-carbonyl]benzenesulfonamide, $\mathbf{5 p}$

Yield: 77\%; M.p.: 272-273 ${ }^{\circ} \mathrm{C}$; $\mathrm{R}_{f}=0.18 .{ }^{1} \mathrm{H}-\mathrm{NMR}$ (DMSO-d $)$ : $(\delta)$ 3.30-3.40 (m, 4H, $\mathrm{CH}_{2}$ ), 3.64$3.74\left(\mathrm{~m}, 4 \mathrm{H}, \mathrm{CH}_{2}\right), 7.46$ (m, 2H, ArH), 7.61-8.29 (m, 8H, ArH). Anal. for $\left(\mathrm{C}_{18} \mathrm{H}_{18} \mathrm{~N}_{4} \mathrm{O}_{6} \mathrm{~S}\right): \mathrm{C} 51.67 \%$, H 4.34\%, N 13.39\%; Found: C 51.77\%, H 4.44\%, N $13.49 \%$.

4-[4-(2-Aminobenzoyl)piperazine-1-carbonyl]benzenesulfonamide, $\mathbf{5 q}$

Yield: 99\%; m.p.: $231-232^{\circ} \mathrm{C}$; $\mathrm{R}_{f}=0.10 .{ }^{1} \mathrm{H}-\mathrm{NMR}$ (DMSO- $\left.d_{6}\right)$ : $(\delta) 3.36-3.76\left(\mathrm{~m}, 8 \mathrm{H}, \mathrm{CH}_{2}\right), 5.20(\mathrm{bs}$, $\left.2 \mathrm{H}, \mathrm{NH}_{2}\right), 6.56$ (t, $\left.J=7.6, J=7.4,1 \mathrm{H}, \mathrm{ArH}\right), 6.70$ (d, $\left.J=8.1,1 \mathrm{H}, \mathrm{ArH}\right), 7.01$ (d, J=7.6, 1H, ArH), 7.08 (t, $J=8.1, J=7.4,1 \mathrm{H}, \mathrm{ArH}), 7.49$ (s, 2H, NH 2$), 7.60$ (d, J=8.0, 2H, ArH ), 7.88 (d, J=8.0, 2H, ArH). Anal. for $\left(\mathrm{C}_{18} \mathrm{H}_{20} \mathrm{~N}_{4} \mathrm{O}_{4} \mathrm{~S}\right)$ : C 55.66\%, H 5.19\%, N 14.42\%; Found: C 55.63\%, H 5.16\%, N $14.39 \%$.

4-[4-(3-Aminobenzoyl)piperazine-1-carbonyl]benzenesulfonamide, $\mathbf{5 r}$

Yield: 87\%; m.p.: 220-223 ${ }^{\circ} \mathrm{C}$; $\mathrm{R}_{f}=0.13 .{ }^{1} \mathrm{H}-\mathrm{NMR}$ (DMSO-d $)$ : $(\delta)$ 3.40-3.66 (m, 8H, $\left.\mathrm{CH}_{2}\right), 5.26(\mathrm{bs}$, $\left.2 \mathrm{H}, \mathrm{NH}_{2}\right), 6.50$ (d, J=7.5, 1H, ArH,), 6.57 (s, 1H, ArH), 6.62 (d, J= 7.5, 1H, ArH, ) 7.07 (t, 1H, ArH, 
$J=7.5), 7.48\left(\mathrm{~s}, 2 \mathrm{H}, \mathrm{NH}_{2}\right), 7.62(\mathrm{~d}, J=8.2,2 \mathrm{H}, \operatorname{ArH}), 7.89(\mathrm{~d}, J=8.2,2 \mathrm{H}, \operatorname{ArH})$. Anal. for $\left(\mathrm{C}_{18} \mathrm{H}_{20} \mathrm{~N}_{4} \mathrm{O}_{4} \mathrm{~S}\right)$ : C 55.66\%, H 5.19\%, N 14.42\%; Found: C 55.63\%, H 5.16\%, N 14.39\%.

\section{4-[4-(4-Aminobenzoyl)piperazine-1-carbonyl]benzenesulfonamide, $\mathbf{5} \mathbf{s}$}

Yield: 50\%; m.p.: $229-230{ }^{\circ} \mathrm{C} ; \mathrm{R}_{f}=0.14 .{ }^{1} \mathrm{H}-\mathrm{NMR}$ (DMSO- $\left.d_{6}\right)$ : $(\delta) 3.49-3.65\left(\mathrm{~m}, 8 \mathrm{H}, \mathrm{CH}_{2}\right.$ ), 5.53 (bs, $2 \mathrm{H}, \mathrm{NH}_{2}$ ), 6.52 (d, J=8.4, 2H, ArH), 7.13 (d, J=8.4, 2H, ArH), 7.46 (s, 2H, NH 2$), 7.59$ (d, J=8.5, 2H, ArH), $7.86(\mathrm{~d}, J=8.5,2 \mathrm{H}, \mathrm{ArH})$. Anal. for $\left(\mathrm{C}_{18} \mathrm{H}_{20} \mathrm{~N}_{4} \mathrm{O}_{4} \mathrm{~S}\right): \mathrm{C} 55.66 \%, \mathrm{H} 5.19 \%, \mathrm{~N} 14.42 \%$; Found: C $55.62 \%, \mathrm{H} 5.15 \%, \mathrm{~N} 14.38 \%$.

\subsection{CA Inhibition Assay}

An Applied Photophysics stopped-flow instrument has been used for assaying the $\mathrm{CA}$ catalysed $\mathrm{CO}_{2}$ hydration activity. Phenol red (at a concentration of $0.2 \mathrm{mM}$ ) has been used as indicator, working at the absorbance maximum of $557 \mathrm{~nm}$, with $10-20 \mathrm{mM}$ Hepes ( $\mathrm{pH} \mathrm{7.5)}$ or Tris (pH 8.3) as buffers, and $20 \mathrm{mM} \mathrm{Na}_{2} \mathrm{SO}_{4}$ or $20 \mathrm{mM} \mathrm{NaClO}_{4}$ (for maintaining constant the ionic strength), following the initial rates of the $\mathrm{CA}$-catalyzed $\mathrm{CO}_{2}$ hydration reaction for a period of 10-100 s. The $\mathrm{CO}_{2}$ concentrations ranged from 1.7 to $17 \mathrm{mM}$ for the determination of the kinetic parameters and inhibition constants. For each inhibitor at least six traces of the initial 5-10\% of the reaction have been used for determining the initial velocity. The uncatalyzed rates were determined in the same manner and subtracted from the total observed rates. Stock solutions of inhibitor (10 mM) were prepared in distilled-deionized water and dilutions up to $0.01 \mathrm{nM}$ were done thereafter with distilleddeionized water. Inhibitor and enzyme solutions were preincubated together for $15 \mathrm{~min}$ at room temperature prior to assay, in order to allow for the formation of the E-I complex. The inhibition constants were obtained by non-linear least-squares methods using PRISM 3, as reported earlier, and represent the mean from at least three different determinations. CA isoforms were recombinant ones obtained as reported earlier by this group..$^{1-3}$

\subsection{X-ray crystallographic studies}

Crystals of the hCA II/5a adduct were obtained by co-crystallization. In detail, the complex was prepared by adding a 5 fold excess of the inhibitor to a $10 \mathrm{mg} / \mathrm{mL}$ protein solution. The mixture was equilibrated for $1 \mathrm{~h}$ at room temperature and used for the crystallization experiments. Drops were prepared by mixing $1 \mu \mathrm{l}$ of hCA II/5a solution with $1 \mu \mathrm{l}$ of precipitant solution containing $1.2 \mathrm{M}$ sodium citrate, $0.1 \mathrm{M}$ Tris- $\mathrm{HCl} \mathrm{pH} 8.5$ and further equilibrated over a well containing $1 \mathrm{~mL}$ of precipitant buffer. Crystals appeared in the drops within 2 days and grew to a maximum dimension of $0.3 \times 0.3 \times 0.2 \mathrm{~mm}^{3}$ in about one week.

X-ray diffraction data were collected at the Elettra Synchrotron Light Source (Trieste) by using one single crystal. Prior to cryogenic cooling, the crystal was transferred into precipitant solution with the addition of $15 \%(\mathrm{v} / \mathrm{v})$ glycerol. Diffraction data were indexed, integrated and scaled using the HKL2000 software package. ${ }^{4}$ Data collection statistics are reported in Table S1.

The initial phases of the structure were calculated using the atomic coordinates of the native hCA II with waters removed. ${ }^{5}$ The structure was refined using the program REFMAC5. $8^{6}$ in CCP $4 \mathrm{i}^{7}$, whereas model building and map inspections were performed with the program O. ${ }^{8}$ Inhibitor topologies and parameters were generated using SKETCHER. ${ }^{9}$ Several rounds of restrained refinement and anisotropic temperature factor refinement alternated with manual rebuilding were necessary to reduce the crystallographic Rwork/Rfree values to $0.135 / 0.146$. Refinement statistics are summarized in 
Table S1. The stereochemical quality of the model was finally checked using Procheck ${ }^{10}$ and Whatcheck ${ }^{11}$ programs.

Coordinates and structure factors have been deposited in the Protein Data Bank (accession code 6XXT).

\subsection{Docking studies}

The crystal structures of hCA XII in complex with the inhibitor acetazolamide was retrieved from the RCSB Protein Data Bank (PDB code 1JD0) ${ }^{12}$ The protein and ligands were prepared by Vega ${ }^{13}$ and used to setup docking simulation by Gold Suite 5.7.1 ${ }^{14}$

The region of interest used by Gold program was defined in order to contain residues within $10 \AA$ from the original position of the ligand in the X-ray structure. A scaffold constraint (penalty = 5.0) was used to restrict the solutions in which the sulfonamide moiety was able to coordinate the metal within the catalytic binding site. ChemPLP was chosen as fitness function and the standard default settings were used in all calculations. Ligands were submitted to 100 genetic algorithm runs and the "allow early termination" command was deactivated. Results differing by less than $0.75 \AA$ An ligandall atom RMSD, were clustered together. The best GOLD-calculated conformation was considered both for analysis and representation.

\section{References}

(1) A. Innocenti, D. Vullo, J. Pastorek, A. Scozzafava, S. Pastorekova, I. Nishimori, C. T. Supuran, Carbonic anhydrase inhibitors. Inhibition of transmembrane isozymes XII (cancer-associated) and XIV with anions. Bioorg. Med. Chem. Lett. 2007, 17 (6), 1532-1537.

(2) I. Nishimori, D. Vullo, A. Innocenti, A. Scozzafava, A. Mastrolorenzo, C. T. Supuran, Carbonic anhydrase inhibitors: inhibition of the transmembrane isozyme XIV with sulfonamides. Bioorg. Med. Chem. Lett. 2005, 15 (17), 3828-3833.

(3) I. Nishimori, D. Vullo, A. Innocenti, A. Scozzafava, A. Mastrolorenzo, C. T. Supuran, Carbonic anhydrase inhibitors. The mitochondrial isozyme VB as a new target for sulfonamide and sulfamate inhibitors. J. Med. Chem. 2005, 48 (24), 7860-7866.

(4) Z. Otwinowski, W. Minor, Processing of X-ray diffraction data collected in oscillation mode. Methods Enzymol 1997, 276, 307-326.

(5) A. E. Eriksson, T. A. Jones, A. Liljas, Refined structure of human carbonic anhydrase II at 2.0 A resolution. Proteins 1988, 4 (4), 274-282.

(6) G. N. Murshudov, P. Skubak, A. A. Lebedev, N. S. Pannu, R. A. Steiner, R. A. Nicholls, M. D. Winn, F. Long, A. A. Vagin, REFMAC5 for the refinement of macromolecular crystal structures. Acta Crystallogr D Biol Crystallogr 2011, 67 (Pt 4), 355-367.

(7) M. D. Winn, C. C. Ballard, K. D. Cowtan, E. J. Dodson, P. Emsley, P. R. Evans, R. M. Keegan, E. B. Krissinel, A. G. Leslie, A. McCoy, S. J. McNicholas, G. N. Murshudov, N. S. Pannu, E. A. Potterton, H. R. Powell, R. J. Read, A. Vagin, K. S. Wilson, Overview of the CCP4 suite and current developments. Acta Crystallogr D Biol Crystallogr 2011, 67 (Pt 4), 235-242.

(8) T. A. Jones, J. Y. Zou, S. W. Cowan, M. Kjeldgaard, Improved Methods for Building Protein Models in Electron-Density Maps and the Location of Errors in These Models. Acta Crystallographica Section A 1991, 47, 110-119.

(9) A. A. Vagin, R. A. Steiner, A. A. Lebedev, L. Potterton, S. McNicholas, F. Long, G. N. Murshudov, REFMAC5 dictionary: organization of prior chemical knowledge and guidelines for its use. Acta Crystallographica Section D-Structural Biology 2004, 60, 2184-2195.

(10) R. A. Laskowski, M. W. Macarthur, D. S. Moss, J. M. Thornton, Procheck - a Program to Check the Stereochemical Quality of Protein Structures. Journal of Applied Crystallography 1993, 26, 283291. 
(11) R. W. W. Hooft, G. Vriend, C. Sander, E. E. Abola, Errors in protein structures. Nature 1996, 381 (6580), 272-272.

(12) D. A. Whittington, A. Waheed, B. Ulmasov, G. N. Shah, J. H. Grubb, W. S. Sly, D. W. Christianson, Crystal structure of the dimeric extracellular domain of human carbonic anhydrase XII, a bitopic membrane protein overexpressed in certain cancer tumor cells. Proc Natl Acad Sci U S A 2001, 98 (17), 9545-9550.

(13) A. Pedretti, L. Villa, G. Vistoli, VEGA: a versatile program to convert, handle and visualize molecular structure on Windows-based PCs. Journal of Molecular Graphics \& Modelling 2002, 21 (1), 47-49.

(14) G. Jones, P. Willett, R. C. Glen, A. R. Leach, R. Taylor, Development and validation of a genetic algorithm for flexible docking. Journal of Molecular Biology 1997, 267 (3), 727-748. 


\section{Selected representative ${ }^{1} \mathrm{H}$ and ${ }^{13} \mathrm{C}$ NMR spectra}

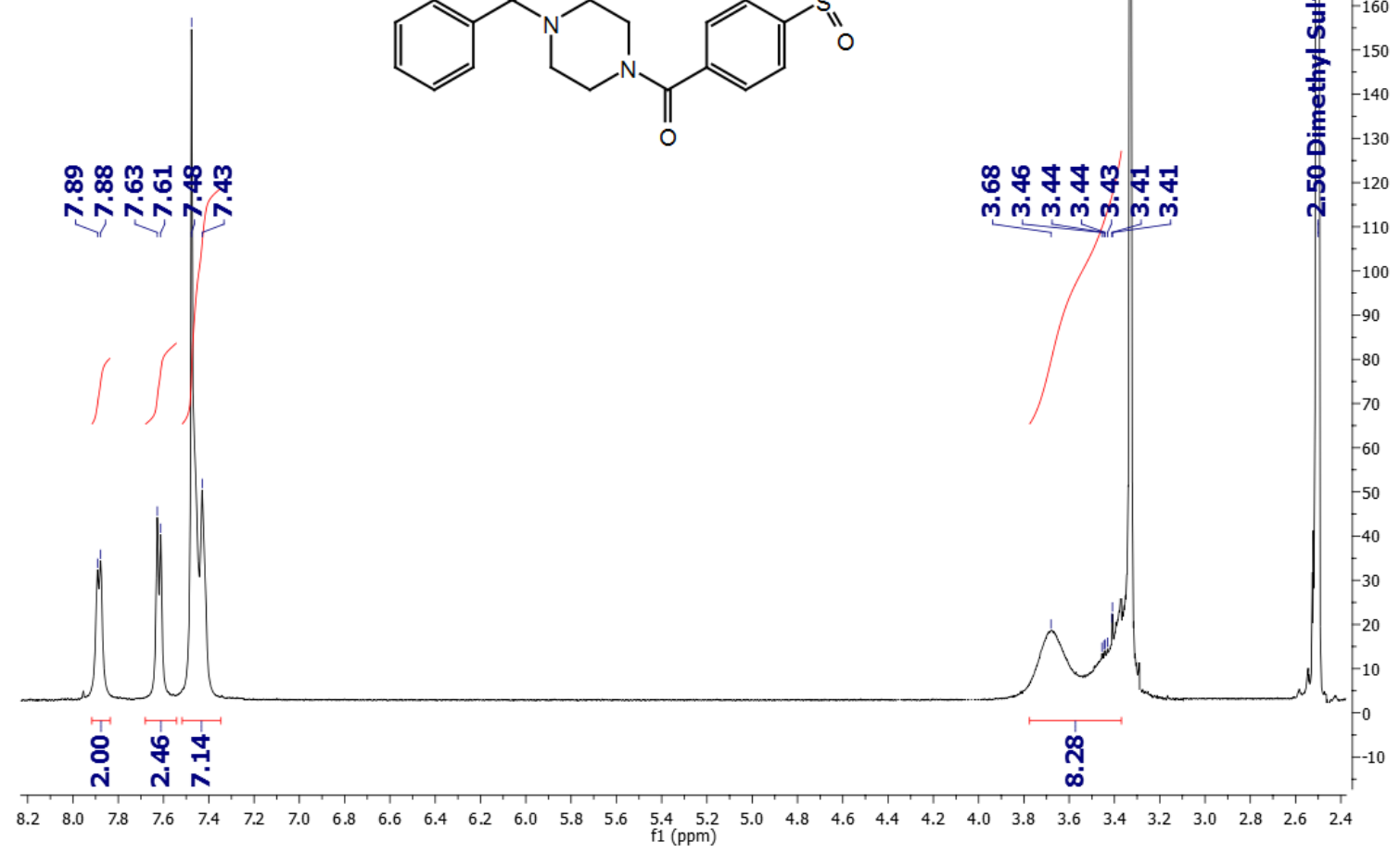

${ }^{1} \mathrm{H}-\mathrm{NMR}\left(\mathrm{DMSO}-\mathrm{d}_{6}\right.$ ) spectrum of 4-[4-(4-Aminobenzoyl)piperazine-1-carbonyl]benzenesulfonamide (5a)

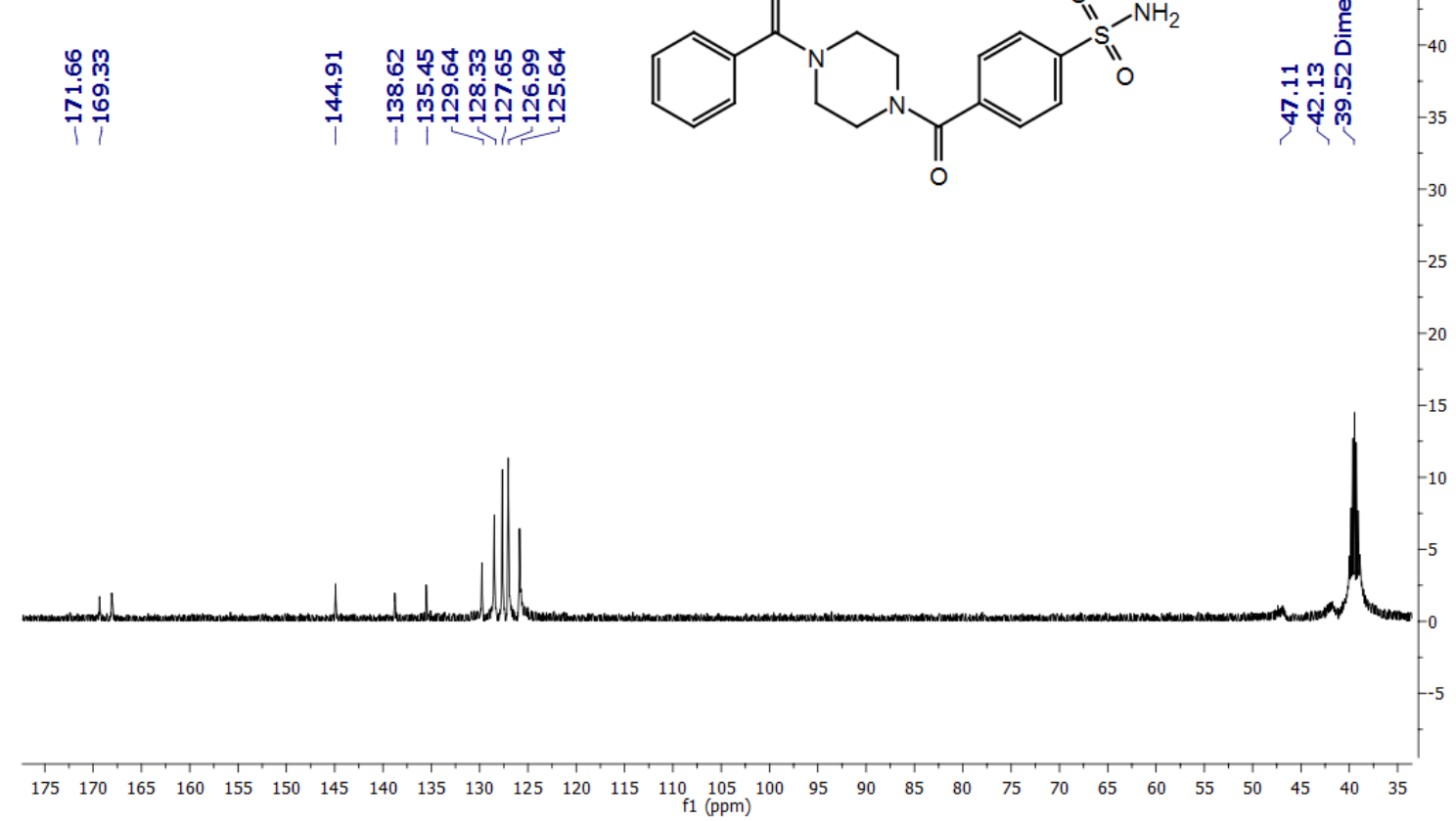

${ }^{13} \mathrm{C}-\mathrm{NMR}$ (DMSO- $d_{6}$ ) spectrum of 4-[4-(4-Aminobenzoyl)piperazine-1-carbonyl]benzenesulfonamide (5a) 


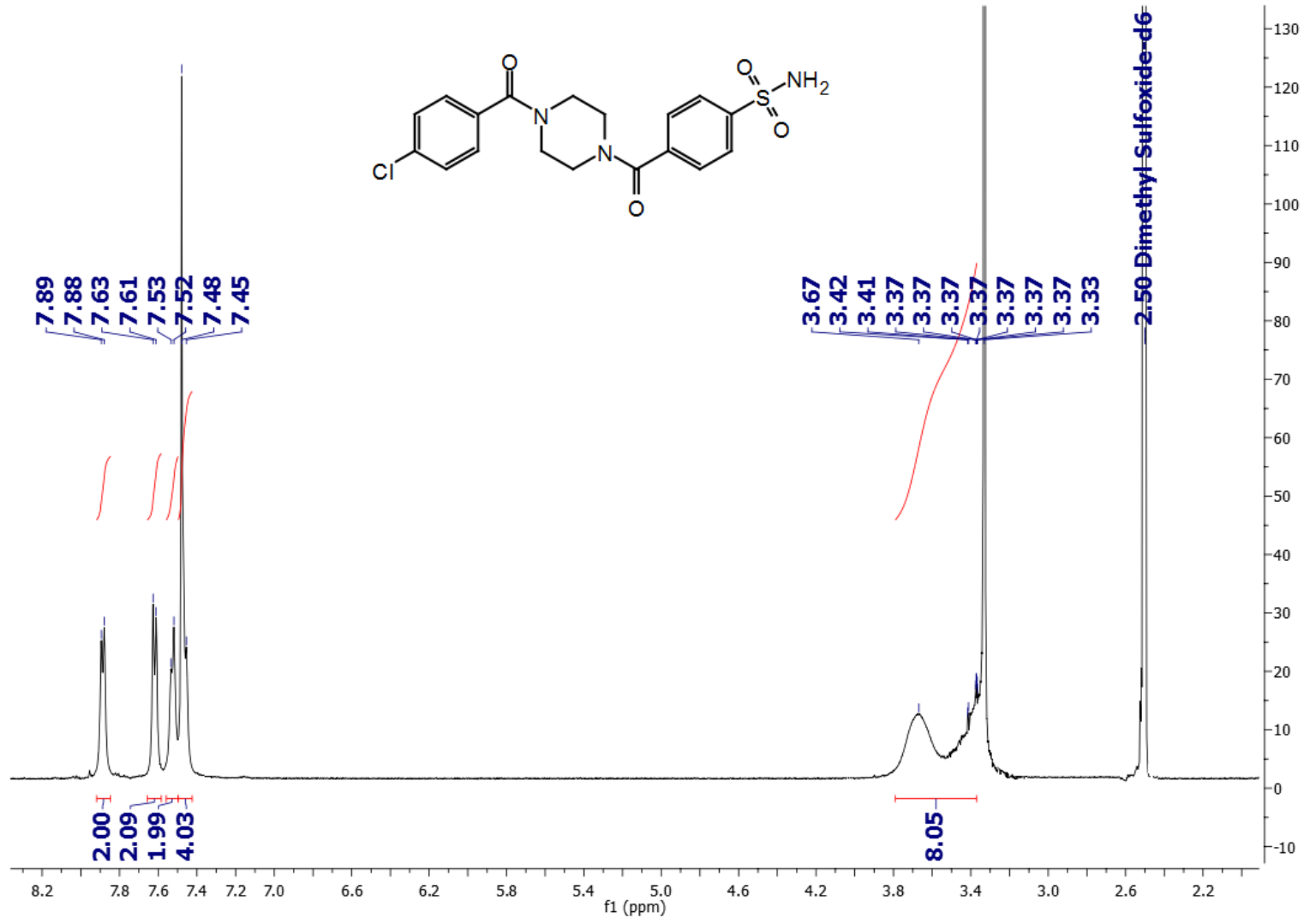

${ }^{1} \mathrm{H}-\mathrm{NMR}\left(\mathrm{DMSO}-\mathrm{d}_{6}\right.$ ) spectrum of 4-[4-(4-Chlorobenzoyl)piperazine-1-carbonyl]benzenesulfonamide (5g)

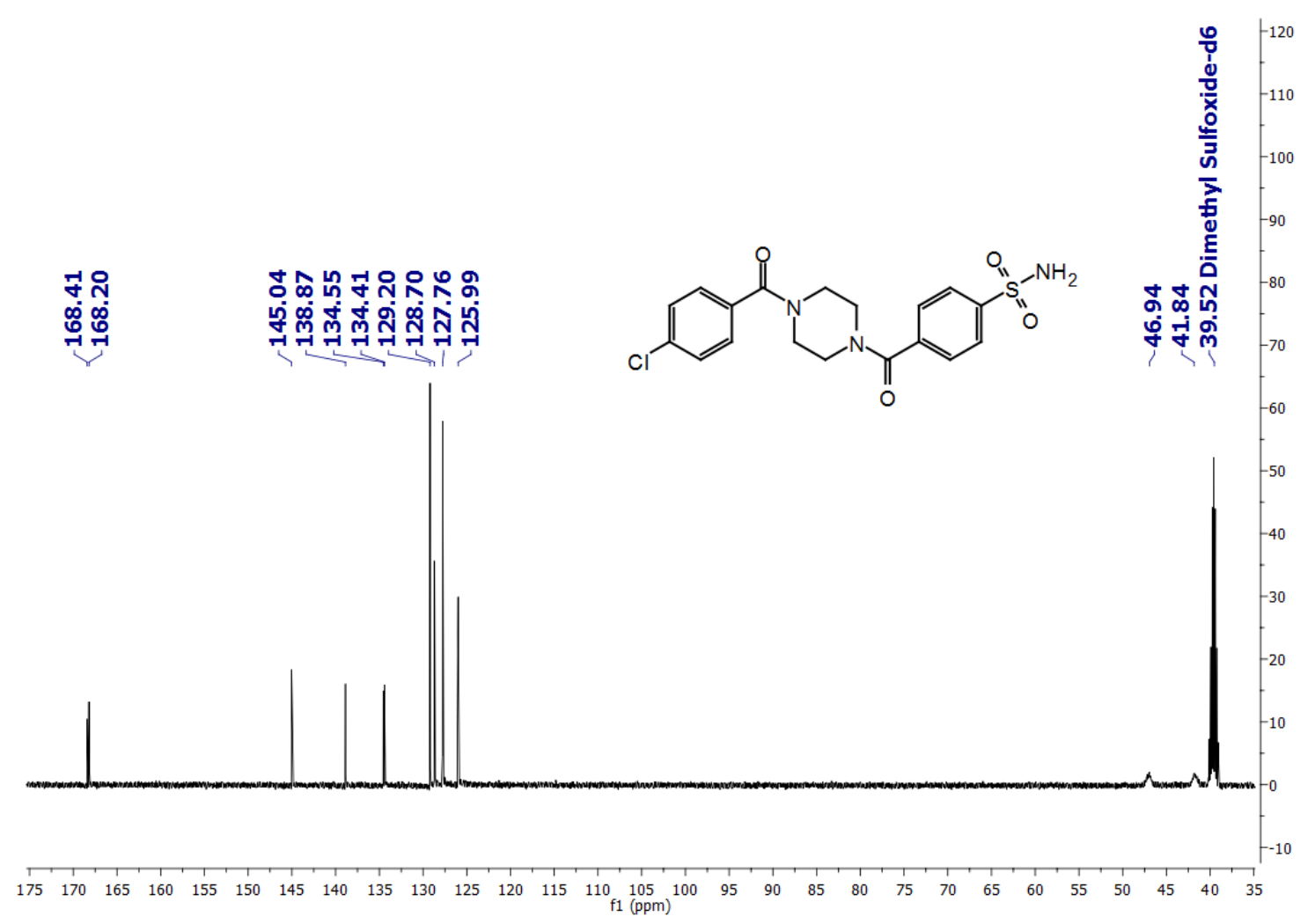

${ }^{13} \mathrm{C}-\mathrm{NMR}\left(\mathrm{DMSO}-d_{6}\right.$ ) spectrum of 4-[4-(4-Chlorobenzoyl)piperazine-1-carbonyl]benzenesulfonamide (5g) 
<smiles>NS(=O)(=O)c1ccc(C(=O)N2CCN(C(=O)c3cccc([N+](=O)[O-])c3)CC2)cc1</smiles>

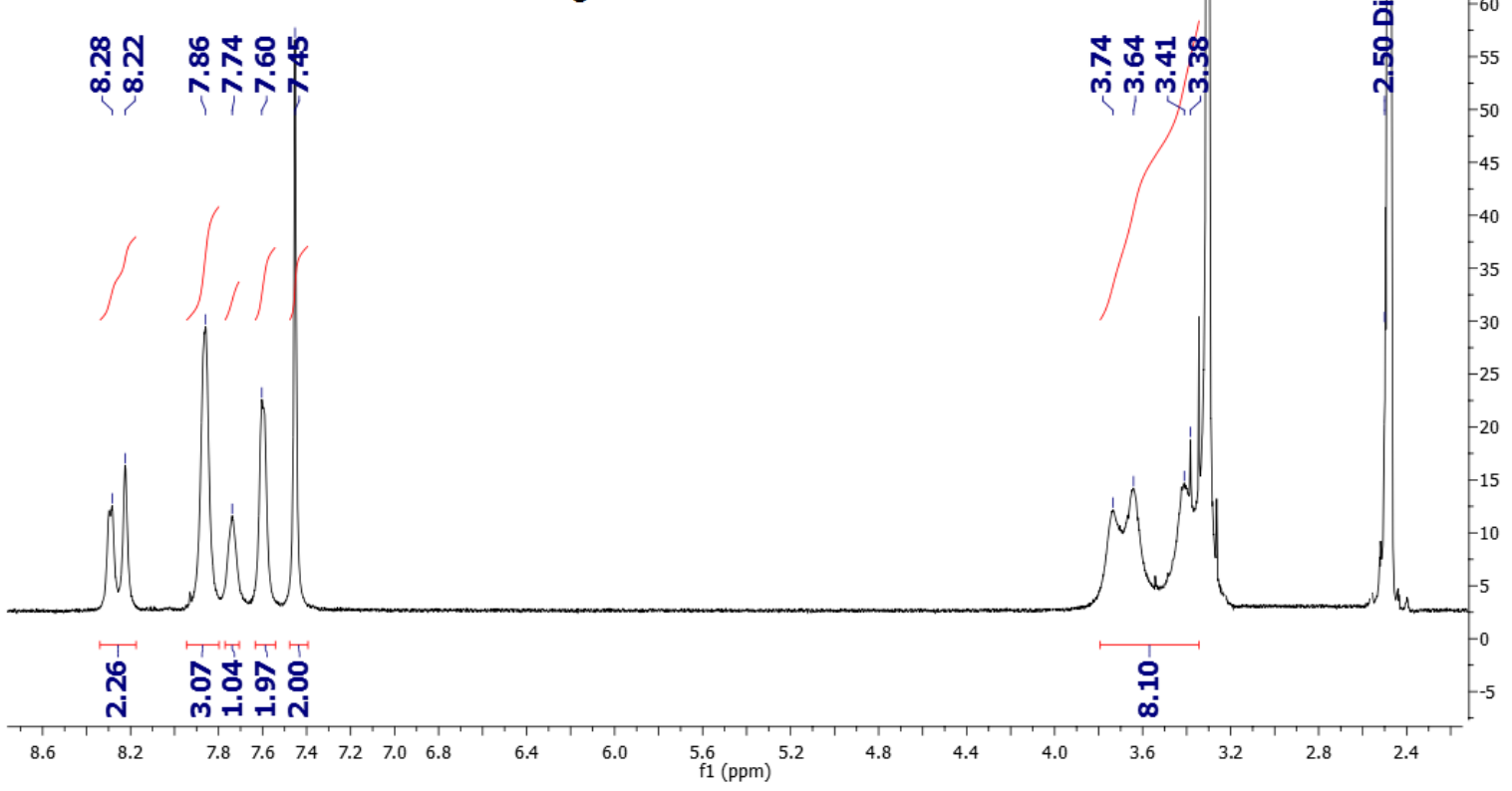

${ }^{1} \mathrm{H}-\mathrm{NMR}\left(\mathrm{DMSO}-d_{6}\right)$ spectrum of 4-[4-(3-Nitrobenzoyl)piperazine-1-carbonyl]benzenesulfonamide (5o)<smiles>Nc1ccccc1C(=O)N1CCN(C(=O)c2ccc(S(N)(=O)=O)cc2)CC1</smiles>

\section{ฌ

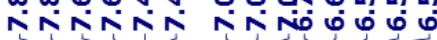

กิำ
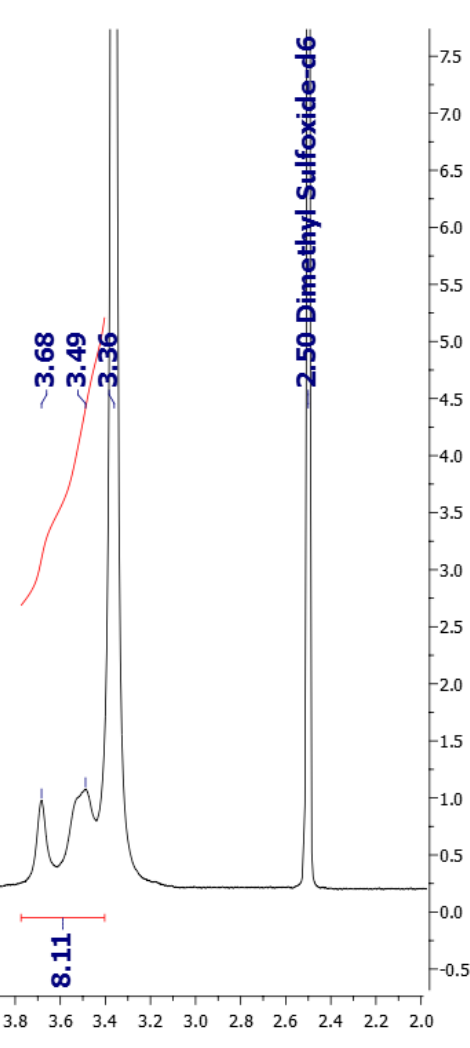

${ }^{1} \mathrm{H}-\mathrm{NMR}\left(\mathrm{DMSO}-d_{6}\right)$ spectrum of 4-[4-(2-Aminobenzoyl)piperazine-1-carbonyl] benzenesulfonamide (5q) 


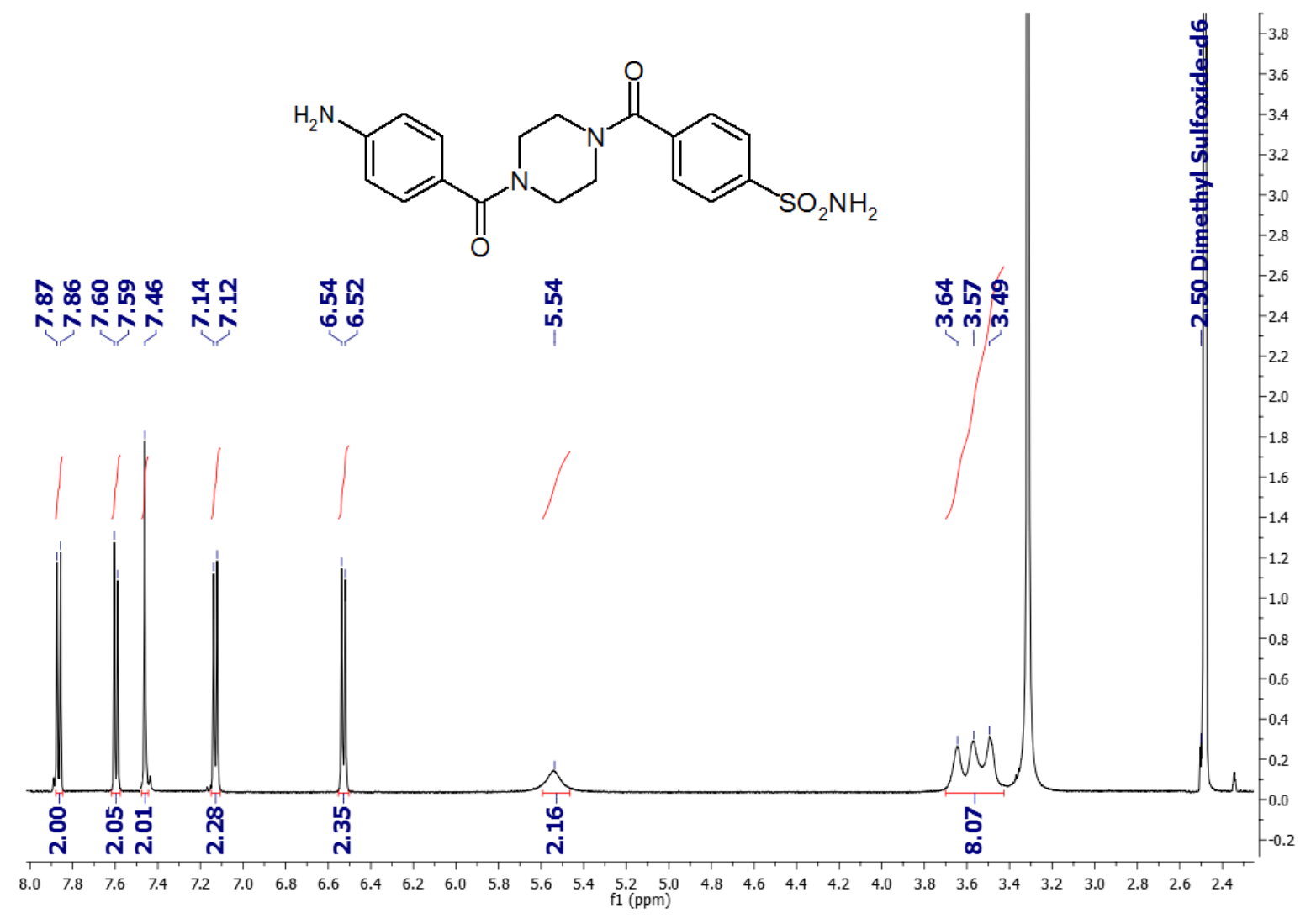

${ }^{1} \mathrm{H}-\mathrm{NMR}$ (DMSO- $d_{6}$ ) spectrum of 4-[4-(4-Aminobenzoyl)piperazine-1-carbonyl]benzenesulfonamide (5s) 


\section{Table S1}

Data collection and refinement statistics for hCA II/5a complex

\begin{tabular}{|c|c|}
\hline & hCA II/5a \\
\hline \multicolumn{2}{|l|}{ Cell parameters } \\
\hline Space group & $\mathrm{P} 2_{1}$ \\
\hline \multirow[t]{4}{*}{ Cell dimensions $\left(\AA,^{\circ}\right)$} & $a=42.3$ \\
\hline & $b=41.5$ \\
\hline & $\mathrm{c}=72.1$ \\
\hline & $\beta=104.3$ \\
\hline Number of independent molecules & 1 \\
\hline \multicolumn{2}{|l|}{ Data collection statistics } \\
\hline Wavelength $(\AA)$ & 1.0 \\
\hline Resolution limits $(\AA)$ & $35.7-1.05$ \\
\hline Total reflections & 652807 \\
\hline Unique reflections & 112517 \\
\hline Redundancy & 5.8 \\
\hline Completeness (\%) & $99.2(86.9)$ \\
\hline R-merge ${ }^{*}$ & $0.072(0.277)$ \\
\hline Rmeas $^{\S}$ & $0.078(0.323)$ \\
\hline Rpim $^{\text {II }}$ & $0.031(0.159)$ \\
\hline$<\mathrm{I}>/<\sigma(\mathrm{I})>$ & $22.1(4.3)$ \\
\hline \multicolumn{2}{|l|}{ Refinement statistics } \\
\hline Resolution limits $(\AA)$ & $35.7-1.05$ \\
\hline R-work $^{* * *}(\%)$ & 13.5 \\
\hline R-free $^{* *}(\%)$ & 14.6 \\
\hline \multicolumn{2}{|l|}{ r.m.s.d. from ideal geometry: } \\
\hline Bond lengths $(\AA)$ & 0.008 \\
\hline Bond angles $\left(^{\circ}\right)$ & 1.5 \\
\hline Number of protein atoms & 2129 \\
\hline Number of inhibitor atoms & 26 \\
\hline Number of water molecules & 271 \\
\hline \multicolumn{2}{|l|}{ Average $\mathrm{B}$ factor $\left(\AA^{2}\right)$} \\
\hline All atoms & 16.03 \\
\hline Protein atoms & 10.54 \\
\hline Inhibitor atoms & 13.49 \\
\hline Waters & 21.36 \\
\hline PDB accession code & $6 \mathrm{XXT}$ \\
\hline
\end{tabular}

$* \mathrm{R}$-merge $=\Sigma_{\mathrm{hk}} \Sigma_{\mathrm{i}}\left|\mathrm{I}_{\mathrm{i}}(\mathrm{hkl})-\langle\mathrm{I}(\mathrm{hkl})\rangle\right| / \Sigma_{\mathrm{hk}} \Sigma_{\mathrm{i}} \mathrm{I}_{\mathrm{i}}(\mathrm{hkl})$, where $\mathrm{I}_{\mathrm{i}}(\mathrm{hkl})$ is the intensity of an observation and $\langle\mathrm{I}(\mathrm{hkl})\rangle$ is the mean value for its unique reflection; summations are over all reflections; $\S R$ meas $=\Sigma_{\mathrm{hkl}}\{\mathrm{N}(\mathrm{hkl}) /[\mathrm{N}(\mathrm{hkl})$ $1]\}^{1 / 2} \mathrm{x} \Sigma_{\mathrm{i}}\left|\mathrm{I}_{\mathrm{i}}(\mathrm{hkl})-<\mathrm{I}(\mathrm{hkl})>\right| / \Sigma_{\mathrm{hkl}} \Sigma_{\mathrm{i}} \mathrm{I}_{\mathrm{i}}(\mathrm{hkl}) ;$ TRpim $=\Sigma_{\mathrm{hkl}}\{1 /[\mathrm{N}(\mathrm{hkl})-1]\}{ }^{1 / 2} \mathrm{x} \Sigma_{\mathrm{i}}\left|\mathrm{I}_{\mathrm{i}}(\mathrm{hkl})-<\mathrm{I}(\mathrm{hkl})>\right| / \Sigma_{\mathrm{hkl}} \Sigma_{\mathrm{i}} \mathrm{I}_{\mathrm{i}}(\mathrm{hkl}) ; * * \mathrm{R}-$ work $=\Sigma_{\text {hkl }}|| \mathrm{Fo}(\mathrm{hkl})|-| \mathrm{Fc}(\mathrm{hkl})|| / \Sigma_{\mathrm{hkl}}|\mathrm{Fo}(\mathrm{hkl})|$ calculated for the working set of reflections. R-free is calculated as for R-work, but from data of the test set that was not used for refinement (Test Set Size $(\%)=1.0)$. Values in parentheses refer to the highest resolution shell (1.07-1.05 $\mathrm{A})$. 